\title{
LAPPED ORTHOGONAL TRANSFORM WITH INTEGER COEFFICIENTS
}

\author{
A.Nallanathan, S.C.Chan and T.S.Ng \\ Department of Electrical and Electronic Engineering. \\ The University of Hong Kong, \\ Pokfulam Road, Hong Kong.
}

\begin{abstract}
In this paper, a new 8-channel integer-valued lapped transform, called the Integer Lapped Orthogonal Transform (ILOT), is proposed. The new transform can be implemented using simple integer arithmetic and has very low implementation complexity. Using 5-bits to represent its transform kemel, it was found that good coding gain and stop band attenuation, similar to the LOT, could be achieved. The multiplier-less fast ILOT requires only 106 additions and 42 shifts and can be impiemented in 32-bit integer arithmetic [13].
\end{abstract}

\section{INTRODUCTION}

Perfect Reconstruction (PR) critically decimated quadrature mirror filter banks (QMFB) have many important applications in speech, audio and image processing. The theory and design of $M$-channel PR maximally decimated uniform filter bank has been exicnsively studied [7]. The cosine modulated filter banks (CMFB) [9] and the lapped transforms [2], [4], [6] are two efficient classes of filter banks with low implementation complexity and good performance. The lapped orthogonal transform (LOT) is an $M$-channel linear-phase PR filter bank with filter length $2 M$. The LOT has slightly higher arithmetic complexity than the DCT but the coding gain is significantly higher with much less blocking artifacts. Recently, there is increasing interest in designing filter banks with low implementation complexity. Approaches based on the sum of power of two (SOPOT) coefficients [3] or integer cocfficients [12] have been proposed. Due to the use of SOPOT, coefficient multiplications can be implemented with simple shifts and additions. In [12], a subspace approach was proposed to design CMFB with integer coefficient prototype filter. Since integer coefficient filter banks require only integer arithmetic (additions and possibly multiplications), the implementation of the filter banks are greatly simplified. Also, if sufficient word length is used to represent the intermediate data, round off error can completely be eliminated.

In this paper, a new lapped orthogonal transform with integer coefficients, called the integer LOT (ILOT) is developed. It can be implemented with simple integer arithmetic and possess very fast implementation algorithm. A multiplier-less implementation has also been developed which requires a total of 106 additions and 42 shifts [13]. Using 5-bits to represent the transform kernel, the ILOT is found to have similar coding gain and image coding performance as the LOT. The paper is organizcd as follows. Section 2 reviews the design of ICT, which was originally proposed by Cham [1]. Construction of the Integer Lapped Orthogonal Transform (ILOT) is described in section 3 . The design and the performance of the ILOT are given in section 4 . Finally, the results are summarized in section 5 , the conclusion.

\section{INTEGER SINUSOIDAL TRANSFORMS}

According to Wang [10], there are four types of DCTs and DST' (type I to IV). DCT-II and DCT-III are the common discrete cosine transform (DCT) and the inverse DCT (IDCT). In this paper, the integer version of the DCT-II (DST-II) and the DCT-IV (DST-IV) are developed to construct the integer lapped orthogonal transform (ILOT). The definition of the DCT-II. DCTIV, DST-II and DST-IV are given as follows,

DCT

DST

$$
\begin{array}{r}
{\left[C_{M}^{\prime \prime}\right]=\sqrt{2 / M}\left\{\varepsilon_{k} \cos (k(n+1 / 2) \pi / M\},\right.} \\
k, n=0,1, \ldots, M-1 . \\
{\left[C_{M}^{N}\right]=\sqrt{2 / M}\left\{\varepsilon_{\alpha} \cos ((k+1 / 2)(n+1 / 2) \pi / M\}\right.} \\
k, n=0,1, \ldots, M-1 .
\end{array}
$$

$$
\begin{array}{r}
{\left[s_{M}^{\prime \prime}\right]=\sqrt{2 / M}\left\{\varepsilon_{k} \sin (k(n-1 / 2) \pi / M\}\right.} \\
k, n=1, \ldots, M . \\
{\left[s_{M}^{N}\right]=\sqrt{2 / M}\left\{\varepsilon_{k} \sin ((k+1 / 2)(n+1 / 2) \pi / M\}\right.} \\
k, n=0,1, \ldots, M-1 .
\end{array}
$$

Where $\varepsilon_{i}=\left\{\begin{array}{cc}1 / \sqrt{2} & i=0 \text { or } M \\ 1 & \text { otherwise }\end{array}\right.$.

Here $[\Lambda]_{i, j}$ represents the $(i, j)$-th element of matrix $A$. The subscript and the superscript of $C$ and $S$ denote, respectively, the length and the type of the transform.

The type-II integer cosine transform (ICT), $T_{\mu}^{C-\| I}$, proposed in [1] has the following matrix form,

$$
P_{M}^{C-t}=K_{M}^{C-\| l} \cdot T_{M}^{C-\| \prime}
$$

where $K_{M}^{C-H}$ is a real diagonal matrix and $P_{M}^{C-u}$ is an orthogonal matrix. The superscript and subscript denote, respectively, the type and the length of the transform. For example, $C-I /$ means the type II cosine transform and so on. Using the concept of dyadic symmetry, Cham [1] constructed an order-8 ICT of the following form. 


$$
T_{z}^{c-a}=\left[\begin{array}{cccccccc}
k & k & k & k & k & k & k & k \\
a & b & c & d & -d & -c & -b & -a \\
e & f & -f & -e & -e & -f & f & e \\
b & -d & -a & -c & c & a & d & -b \\
k & -k & -k & k & k & -k & -k & k \\
c & -a & d & b & -b & -d & a & -c \\
f & -e & e & -f & -f & e & -e & f \\
d & -c & b & -a & a & -b & c & -d
\end{array}\right]
$$

To satisfy the orthogonality properties, $a, b, c$, and $d$ must satisfy the following condition,

$$
a \cdot b=a \cdot c+b \cdot d+c \cdot d \text {. }
$$

Also, in order to make the basis vectors of the ICT resemble those of the $\mathrm{DCT}-\mathrm{Il}, a, b, c, d, e$, and $f$ must satisfy the conditions.

$$
a \geq b \geq c \geq d \text { and } e \geq f \text {. }
$$

In [1], the constants $a, b, c, d, e, f$, and $k$ are chosen to be 8 -bit integers. The $(i, j)$-th entry of matrix $K_{d}^{i-1}, k_{i}$, is used to normalize the $i-$ th row of $T_{M}^{-i t}$ so that $P_{M}^{c-\nu}$ is orthogonal. If $T_{r}$ is the $L_{2}$-norm of the $i$-th row of $T_{M}^{C-1 t}$. then

$$
k_{i}=\left|1 / T_{i}\right| \text {. }
$$

An exhaustive search is then perfomed to maximize the coding gain for common AR(1) process. The multiplication with $k_{i}$ 's are ustally absorbed into the quantization process to reduce the complexity. The inverse of the ICT is given by.

$$
\begin{aligned}
& \left(P_{M}^{C-n}\right)^{-1}=\left(P_{M}^{C \cdots n}\right)^{T}=\left(K_{N}^{C-n} \cdot T_{w}^{C-U}\right)^{r} \\
& =\left(T_{*}^{c-n}\right)^{r}\left(K_{\mu}^{c-n}\right)^{r}
\end{aligned}
$$

The scaling $\left(K_{i}^{-t}\right)^{T}$ can also be absorbed into the dequantization process.

\section{INTEGER LOT (ILOT)}

The transform matrix of an $M$-channel LOT is given by [2].

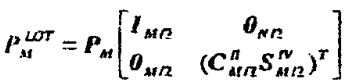

$$
\begin{aligned}
& {\left[\begin{array}{cc}
D_{c}-D_{0} & J_{A 1 / 2}\left(D_{c}-D_{o}\right) \\
D_{c}-D_{\alpha} & -J_{A 1 / 2}\left(D_{c}-D_{v}\right)
\end{array}\right] .}
\end{aligned}
$$

where $D$, and $D_{0}$ are $(M / 2 \times M)$ matrices containing the even and odd basis functions of DCT-H, respectively. $P_{4}$ is a permutation matrix which permules the $k$ th and the $(k+M / 2)$ th rows to the $2 k$ th and the $(2 k+1)$ th ( $k=0, \cdots \cdots, M / 2-1$ ) rows, respectively. Fig. 1 shows the flow graph of an 8-channel LOT. It is natural to consider replacing the 8-point DCT in the LOT by $P_{8}^{C-u}$ to construct an integer LOT. Unfortunately, the scaling matrix. $K_{z}^{c-n}$, is real-valued. Therefore, it is necessary to perform real multiplications immediately after $T_{\mathrm{B}}^{i-n}$ before subsequent additions in the LOT can be performed. A method to avoid this problem is to design an integer cosine transform with all the scalings being identical. In this case, the scaling constant can be moved to the end of the transformation and absorbed in the quantization proxess. The remaining problem is to find similar integer transform for the 4-point DCT-II and the 4-point DST-IV. Using the same concept in [1], we propose the following form for the order 4 type-1l integer cosine transform

$$
T_{4}^{C-u}=\left[\begin{array}{cccc}
\ell & \ell & \ell & \ell \\
a_{1} & b_{1} & -b_{1} & -a_{1} \\
\ell & -\ell & -\ell & \ell \\
b_{1} & -a_{1} & a_{1} & -b_{1}
\end{array}\right],
$$

where $a_{1}, b_{1}$, and $\ell$ are integers to be determined. Like $T_{\mathrm{g}}^{c-1 \prime}, a_{1}$ and $b_{1}$ have to satisfy.

$$
a_{1} \geq b_{1} \text {. }
$$

Similarly, the order-4 type-IV integer sine transform is defined as follows.

$$
T_{1}^{s-w}=\left[\begin{array}{cccc}
a_{2} & b_{2} & c_{2} & d_{2} \\
b_{2} & d_{2} & a_{2} & -c_{2} \\
c_{3} & a_{2} & -d_{2} & b_{2} \\
d_{2} & -c_{2} & b_{2} & -a_{2}
\end{array}\right] .
$$

To satisfy the crthogonal properties, $a_{2}, b_{2}, c_{2}, d_{2}$ must satisfy the following condition,

$$
c_{2} \cdot d_{2}=a_{3} \cdot b_{2}+b_{2} \cdot d_{3}+c_{2} \cdot a_{2} \text {. }
$$

In addition, they have to satisfy the following condition to rescmble the DCT-IV.

$$
a_{2} \geq b_{2} \geq c_{3} \geq d_{1} \text {. }
$$

For simplicity the scaling constants for the matrix product. $\left(T_{4}^{c-11} T_{4}^{5-1 v}\right)^{T}$, are designed to be identical. Finally, the tranisform matrix of the order-8 ILOT is obtained as follows.

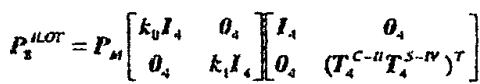

$$
\begin{aligned}
& {\left[\begin{array}{cc}
\tilde{D}_{,}-\tilde{D}_{0} & J_{\alpha}\left(\tilde{D}_{,}-\tilde{D}_{a}\right) \\
\tilde{D}_{c}-\tilde{D}_{c} & -J_{4}\left(\bar{D},-\tilde{D}_{a}\right)
\end{array}\right] \text {. }}
\end{aligned}
$$

where $\bar{D}$, and $\tilde{D}_{a,}$ are $(4 \times 8)$ matrices containing the even and odd basis functions of $T_{\mathrm{s}}^{c-4}$, respectively. The scaling constant for $T_{\mathrm{s}}^{c-u}$ is $k_{0}$. For odd-indexed outputs, the scaling of $T_{3}^{c-u r}$ and $\left(T_{4}^{C-\pi} T_{4}^{S-N}\right)^{T}$ are merged together to form $k_{1}$. These multiplications can readily be absorbed into the: quantization process. Fig. 2 shows the flow graph of the 8-channel integer LOT. The ILOT is parameterized by a set of integers and is referred to as $\operatorname{ILOT}\left(a, b, c, d, e, f, k, a_{1}, b_{1}, \ell, a_{2}, b_{2}, c_{2}, d_{2}\right)$. As an example, $\quad$ ILOT $(24,20,12,6,23,7,17,17,7,13,3,6,10,12)$ refers to the 8-channel length-16 ILOT with $a=24, b=20, c:=12, d=6, e=23, f=7, k=17, a_{1}=17$. $b_{1}=7, l=13, a_{2}=3, b_{2}=6, c_{2}=10$ and $d_{2}=12$.

\section{DESIGN OF THE ILOT}


We now proceed to the design of the ILOT. An important issue in transform and subband coding is the efficiency of the transform or filter bank employed. The coding gain is frequently used as an effective measure of transform efficiency. For orthogonal transform, the coding gain, $G_{T C}$, is given by:

$$
G_{T}=\frac{\frac{1}{M} \sum_{i=0}^{H-1} \sigma_{i}^{2}}{\left(\prod_{i=0}^{N-1} \sigma_{i}^{2}\right)},
$$

where $\sigma_{i}^{2}$ is the variance of the output at the $i$-th analysis filter. For subband coding, the general coding gain formula is given by [11],

$$
G_{S B C}=\frac{1}{M\left(\prod_{i=0}^{M-1} A_{k} B_{k}\right)^{1 / 19}},
$$

$A_{k}=\sum_{j=0}^{N-1} \sum_{i=0}^{N-1} h_{k}(j) h_{k}(i) \rho^{|i-1|}$ and $B_{k}=\frac{1}{M} \sum_{j=0}^{N-1} g_{k}^{3}(j)$.

$h_{k}(n)$ and $g_{k}(n)$ are the impulse responses of the $k$-th analysis and synthesis filters of length $N$. Here the input is assumed to be a first-order auto-regressive process with correlation coefficient $\rho$.

To reduce the wordlength for the integer implementation, the kernel for $T_{s}^{C-l I}, T_{4}^{C-\|}$ and $T_{4}^{S-n}$ are chosen to be 5-bits. Exhaustive search is performed to optimize the coding gain of this ILOT and the optima solution is $\operatorname{LOT}(24,20,12,6,23,7,17,17,7,13,3,6,10,12)$. The coding gain of this ILOT is $9.16 \mathrm{~dB}$ for AR(1) process with correlation coefficient of 0.95 . This is close to the coding gain of the LOT, which is $9.22 \mathrm{~dB}$. Fig. 3a and Fig. $3 \mathrm{~b}$ show respectively the frequency responses of the $\operatorname{ILOT}(24,20,12,6,23,7,17,17,7,13,3,6,10,12)$ and the LOT. The diagonal scaling values $k_{0}$ and $k_{1}$ are 0.0104 and 0.00002353 , respectively. A multiplier-less implementation of this ILOT has also been developed in [13] which requires 106 additions and 42 shifts. Image coding experiments also confirm that its performance is very close to the LOT[13].

\section{CONCLUSION}

In this paper, a new 8-channel integer-valued lapped transform, called the Integer Lapped Orthogonal Transform (ILOT), is presented. The new transform can be implemented using simple integer arithmetic and has very low implementation complexity. Using 5-bits to represent its transform kernel, it was found that good coding gain and stop band attenuation. similar to the LOT, could be achieved. The multiplier-less fast ILOT requires only 106 additions and 42 shifts and can be implemented in 32-bit integer arithmetic [13].

\section{REFERENCES}

1. W. K. Cham, "Development of integer cosine transforms by the principle of dyadic symmetry". Proc. Inst. Elect.Eng. Pt.I, pp. 276-282, Aug 1989.

2. H. S. Malvar and D. H. Staelin, "The LOT: Transform coding without blocking effects", IEEE Trans ASSP-37, pp. 553-559, April 1989.

3. B. R. Horng, H. Samueli and A. N. Willson, "The design of Two-Channel Latlice-Structure PerfectReconstruction Filter banks Using Powers-of-Two Coefficients"' IEEE Trans CAS Vol 40, No 7, July 1993.

4. S. C. Chan, A.Nallanathan, T.S.Ng and P.C.K.Kwok, "A Class of M-Channel Linear-Phase Biorthogonal Filter Banks and Their Applications to Subband Coding" IEEE Trans on Signal Processing, Vol.47, No.2, pp 564-571, February 1999.

5. S. C. Chan and K. L. Ho, "fast algorithms for computing the dicrete cosine transform", IEEE Trans CAS-39, pp. 185-190, March 1992.

6. S. C. Chan, "The generalized lapped transform (GLT) for subband coding applications", in Proc ICASSP' 95, pp. 1508-1511, 1995, Detroit, USA.

7. P. P. Vaidyanathan, Multirate systems and Filter banks, Engltwood Cliffs: Printice-Hall, 1993.

8. T. Q. Nguyen and P. P. Vaidyanathan, "Two-channel perfect reconstruction FIR QMF structures which yicld linear phase PR analysis and synthesis filters." IEEE Trans ASSP, vol ASSP-37, pp. 676-690, May, 1989.

9. R. D. Koilpillai and P. P. Vaidyanathan, "Cosinemodulated FIR filter banks satisfying perfect reconstruction" IEEE Trans. On SP, pp 770-783, Apr. 1992.

10. Z.Wang, "Fast algorithms for the discrete W transform and for the discrete Fourier transform", IEEE Trans., ASSP-32, pp. 803-816, August 1984.

11. J. Katto and Y. Yasuda, "Performance evaluation of subband coding and optimization of its filter coefficients" in Proc. SPIE's Visual Communications and Image processing. pp. 95-106, November 1991.

12. A.Mertins "Subspace Approach for the Design of Cosine-Modulated Filter Banks with Linear-Phase Prototype Filter," IEEE Trans on Signal Processing, Vol. 46, No. 10, October 1998.

13. W. C. Fong, S. C. Chan and A. Nallanathan, and K. L. Ho," Integer lapped transforms and their applications to image coding". Intemal Report, Dept of EEE, The University of Hong Kong, September 1998. 


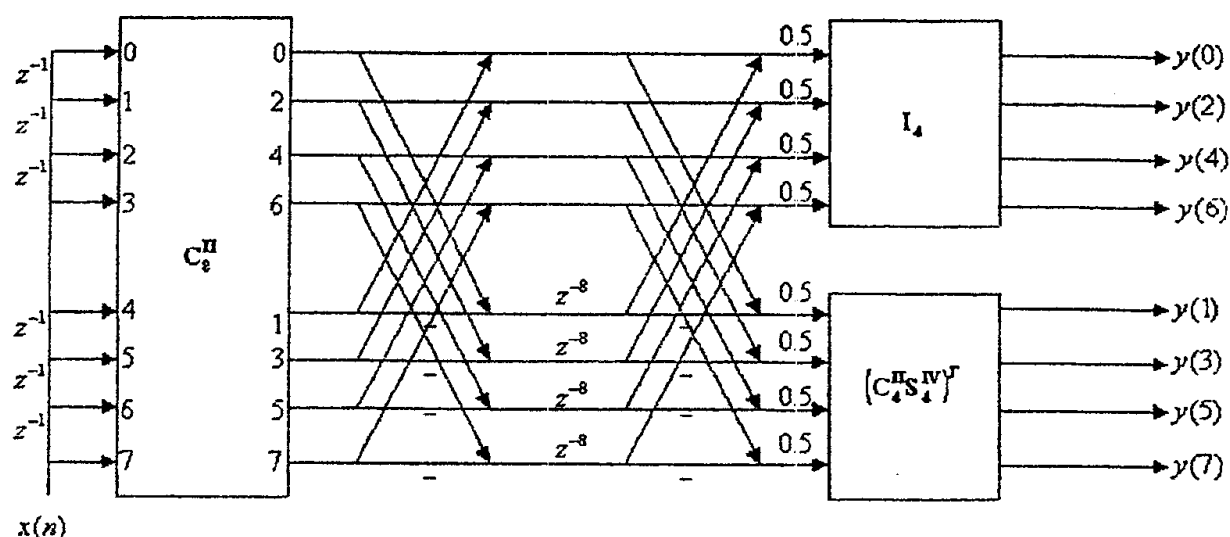

Fig.1 Flow graph of 8-channel LO]?

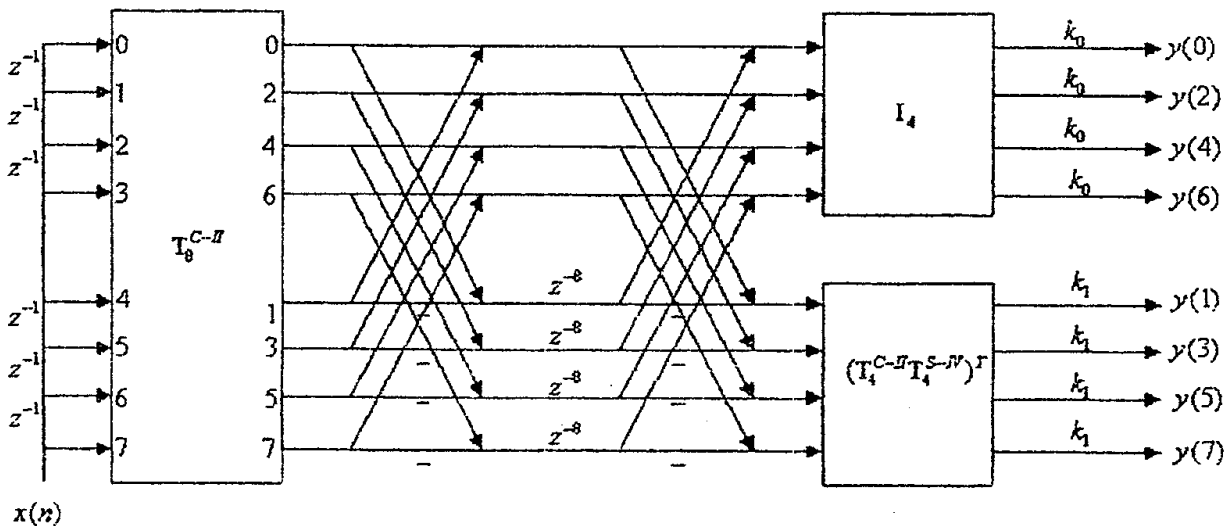

Fig. 2 Flow graph of 8-channel ILOT

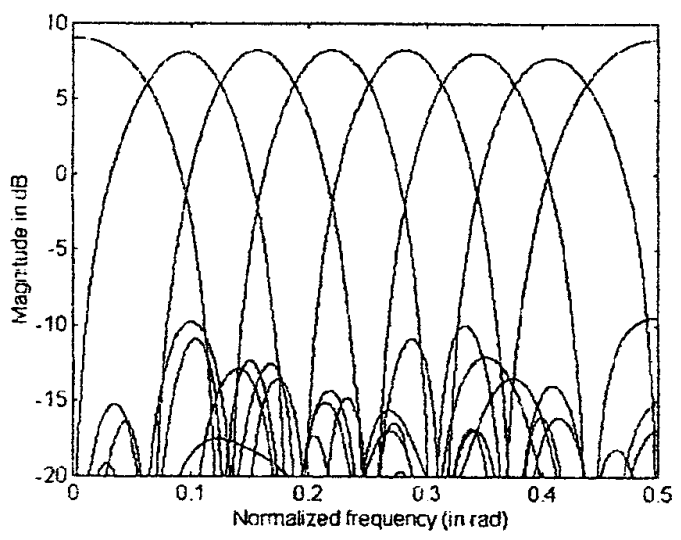

Fig. 3a Frequency response of 8-channel ILOT

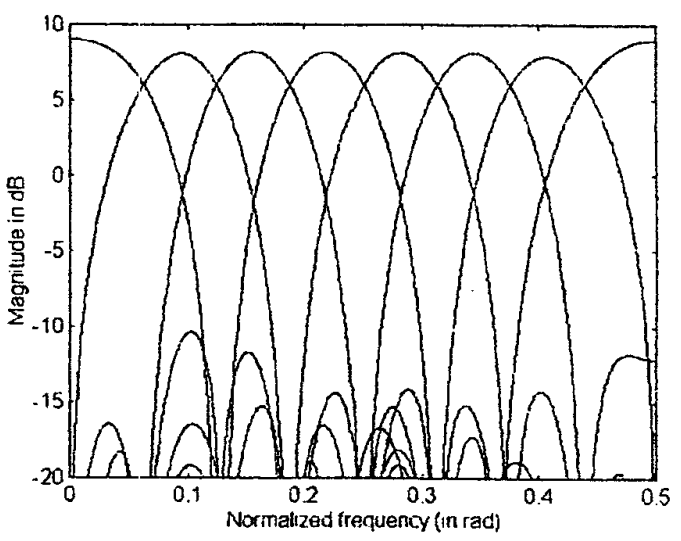

Fig. 3b Frequency response of 8-channel LOT 